\title{
Slowly Progressive Male Alport Syndrome Evaluated by Serial Biopsy: Importance of Type IV Collagen Staining
}

\author{
Masayo Sato ${ }^{1}$, Shun Manabe ${ }^{1}$, Mitsuyo Itabashi ${ }^{1}$, Shigeru Horita ${ }^{2}$, Orie Hirose ${ }^{3}$, \\ Moe Kawashima ${ }^{1}$, Miki Nishida ${ }^{1}$, Hiroshi Kataoka ${ }^{1,4}$, Sekiko Taneda ${ }^{3}$, Toshio Mochizuki ${ }^{1,4}$ \\ and Kosaku Nitta ${ }^{1}$
}

\begin{abstract}
:
A slowly progressive middle-aged man initially diagnosed with thin basement membrane nephropathy based on extensive thinning of the glomerular basement membrane (GBM) was subsequently diagnosed with Alport syndrome (AS) by a serial renal biopsy eight years later. The ultrastructural analysis of the second biopsy indicated thickening and wrinkling with mild reticulation in the GBM, consistent with AS. However, a retrospective analysis of the first biopsy revealed mild attenuation of type IV collagen $\alpha 5$ chain staining, suggesting a potential diagnosis of AS, despite the lack of ultrastructural features of AS. We herein report the clinical usefulness of type IV collagen staining in the early diagnosis of AS.
\end{abstract}

Key words: Alport syndrome, renal biopsy, thin basement membrane nephropathy, type IV collagen

(Intern Med Advance Publication)

(DOI: 10.2169/internalmedicine.7372-21)

\section{Introduction}

Alport syndrome (AS) is a progressive hereditary glomerular disease that results in end-stage renal disease. AS can be associated with hearing loss and eye lesions (1). In contrast to AS, thin basement membrane nephropathy (TBMN) is a benign disease that does not result in an impaired renal function. Therefore, AS should be differentiated from TBMN in practice (2).

Two types of AS have been identified: X-linked AS (XLAS), caused by a mutation on the type IV collagen $\alpha 5$ chain (COL4A5); and autosomal recessive AS (ARAS) or autosomal dominant AS (ADAS), caused by a mutation on the type IV collagen $\alpha 3$ (COL4A3) or $\alpha 4$ chain (COL4A4). Each type of AS has a different prognosis, with a slower disease progression for ADAS than XLAS in men and than ARAS regardless of sex. By comparison, TBMN is defined by thinning observed in $\geq 50 \%$ of glomerular basement membranes (GBMs), with the absence of any GBM thicken- ing, wrinkling, reticulation and lamellation (3). Although TBMN generally has a good prognosis, poor prognostic outcomes have recently been reported for cases originally assumed to be TBMN (4). These cases are often diagnosed later as ADAS (5). As such, the early differentiation of TBMN from AS is important but often not straightforward.

We herein report a patient in whom the observation of thinning in the GBM led to a diagnosis of TBMN on a first renal biopsy. However, a subsequent renal biopsy, performed due to exacerbation of urinary findings over a period of eight years, confirmed a diagnosis of AS based on ultrastructural abnormalities in the GBM. Retrospective analyses of the first biopsy specimen, including type IV collagen staining, led to an early diagnosis of AS. Based on our experience, we propose that type IV collagen staining might be useful for the early diagnosis of atypical AS.

\section{Case Report}

The patient was a 49-year-old Japanese man. His mother

\footnotetext{
${ }^{1}$ Department of Nephrology, Tokyo Women's Medical University, Japan, ${ }^{2}$ Department of Clinical Laboratory Medicine, Tokyo Women's Medical University Hospital, Japan, ${ }^{3}$ Department of Pathology, Tokyo Women's Medical University, Japan and ${ }^{4}$ Clinical Research Division for Polycystic Kidney Disease, Department of Medicine, Kidney Center, Tokyo Women's Medical University, Japan

Received: February 18, 2021; Accepted: August 29, 2021; Advance Publication by J-STAGE: October 12, 2021

Correspondence to Dr. Toshio Mochizuki, mtoshi@twmu.ac.jp
} 

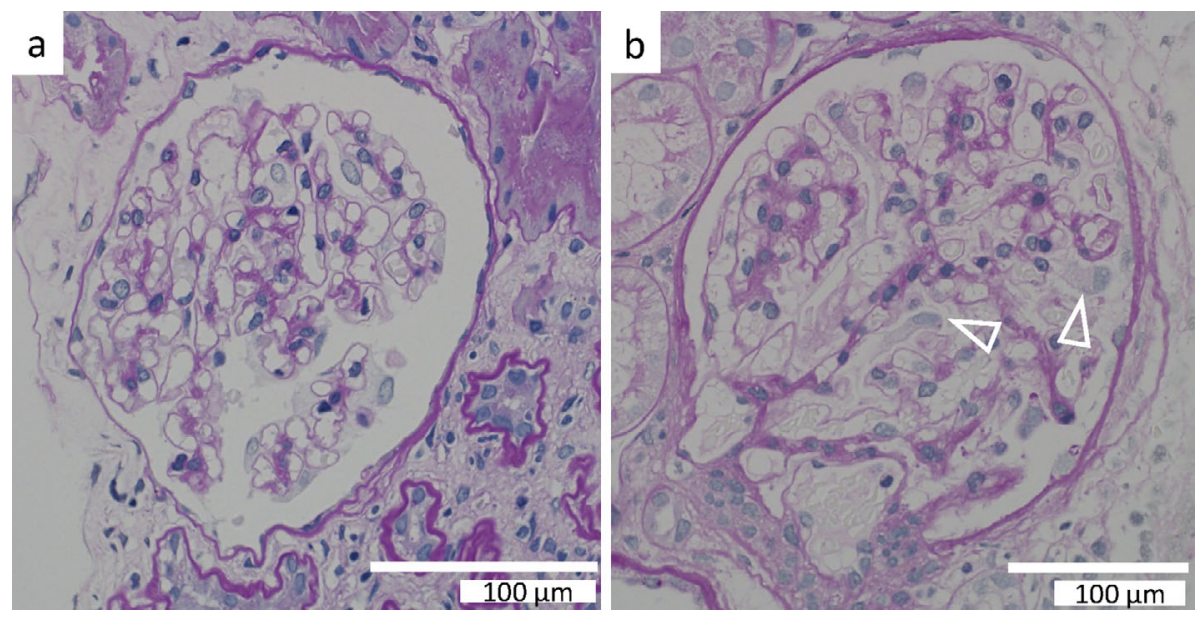

Figure 1. Light microscopy findings for the first (a) and second (b) renal biopsy specimens. (a) No proliferative or sclerotic changes are observed in the glomeruli (PAS stain, $\times 400$ ). (b) The glomeruli are slightly hypertrophic with swelling of the podocytes (arrowhead) (PAS stain, $\times \mathbf{4 0 0}$ ).

had childhood hearing loss, but no hematuria and no renal dysfunction were identified. His older brother had had hematuria since childhood and a decreased renal function (eGFR $15 \mathrm{~mL} / \mathrm{min} / 1.73 \mathrm{~m}^{2}$ ) without hearing loss or fleck retinopathy. The patient had experienced hematuria since childhood, with proteinuria identified at 31 years old. Both hematuria and proteinuria persisted thereafter, and the patient was referred to our department at 41 years old.

A clinical examination revealed proteinuria $(0.46 \mathrm{~g} / \mathrm{gCr})$, hematuria, and an estimated glomerular filtration rate (eGFR) of $74.1 \mathrm{~mL} / \mathrm{min} / 1.73 \mathrm{~m}^{2}$ with hypertension and hyperuricemia. TBMN was diagnosed based on the findings of the first renal biopsy (Fig. 1a, 2a and 2b). After the first biopsy, several anti-hypertensive agents and diuretics were administered, and urate-lowering agents and lipid lowering agents were added (Supplementary (Fig. 1). At 48 years old, urinary protein increased $(2 \mathrm{~g} / \mathrm{gCr})$, and the eGFR decreased $\left(50.5 \mathrm{~mL} / \mathrm{min} / 1.73 \mathrm{~m}^{2}\right)$. The second renal biopsy was performed at 49 years old, leading to a revised diagnosis of AS (Fig. 1b, 2c, and 2d).

\section{Findings on the first renal biopsy}

On light microscopy, 5 of 25 glomeruli showed global sclerosis, and 1 had adhesions (Fig. 1a). Electron microscopy findings were suggestive of TBMN, namely GBM thinning and no evidence of AS, such as thickening of the GBM (Fig. 2a and 2b).

\section{Findings on the second renal biopsy}

On light microscopy, global sclerosis in 1 out of 10 glomeruli, 1 periglomerular fibrosis, and adhesions were observed. The glomeruli were slightly hypertrophic, with swelling of the podocytes (Fig. 1b), and showed mild interstitial fibrosis and tubular atrophy. Although GBM thinning was the main presenting feature on electron microscopy, thickening, wrinkling, collapse (Fig. 2c), and irregularity (Fig. 2d) of the lamella densa in the GBM, findings suggestive of AS, were observed.

\section{Type IV collagen staining}

Retrospective analyses of the first biopsy specimen were performed. Type IV collagen staining revealed partial attenuation of type IV collagen $\alpha 5$ chain ( $\alpha 5[\mathrm{IV}])$ in the GBM, consistent with AS (Fig. 3d), with $\alpha 2$ (IV) staining restricted to the mesangial region (Fig. 3e). In the second renal biopsy specimen, attenuation of $\alpha 5$ (IV) staining in the GBM was more noticeable (Fig. $3 \mathrm{~g}$ ), and $\alpha 2$ (IV) staining was positive along the GBM in addition to the mesangial region (Fig. 3h) compared with the first biopsy (Fig. 3d and 3e).

\section{Genetic analyses}

We performed genetic analyses via next-generation sequencing using targeting hybrid-capture methods that covered all of the exon and the adjacent introns within 10 bases. However, no mutations were identified in either $C O L$ 4A3, COLAA4, or COLAA5.

\section{Discussion}

We herein report a slowly progressive man with AS evaluated by a serial biopsy. Although ultrastructural features of AS were not observed on the first biopsy, attenuation of $\alpha 5$ (IV) staining in the GBM was observed, indicating a diagnosis of AS. Our findings underscore the potential utility of type IV collagen staining when AS is suspected in cases of TBMN.

No mutation was identified in either COLAA3, COLAA4, or COLAA5. For XLAS, approximately $10 \%$ of patients with no identified mutation have been described in the literature (6). There is a possibility of deep intronic mutations (7). Based on the histological findings of electronic microscopy and type IV collagen staining on the second biopsy, the patient had a potential diagnosis of XLAS with a mild genotype but not with a truncating mutation. However, 

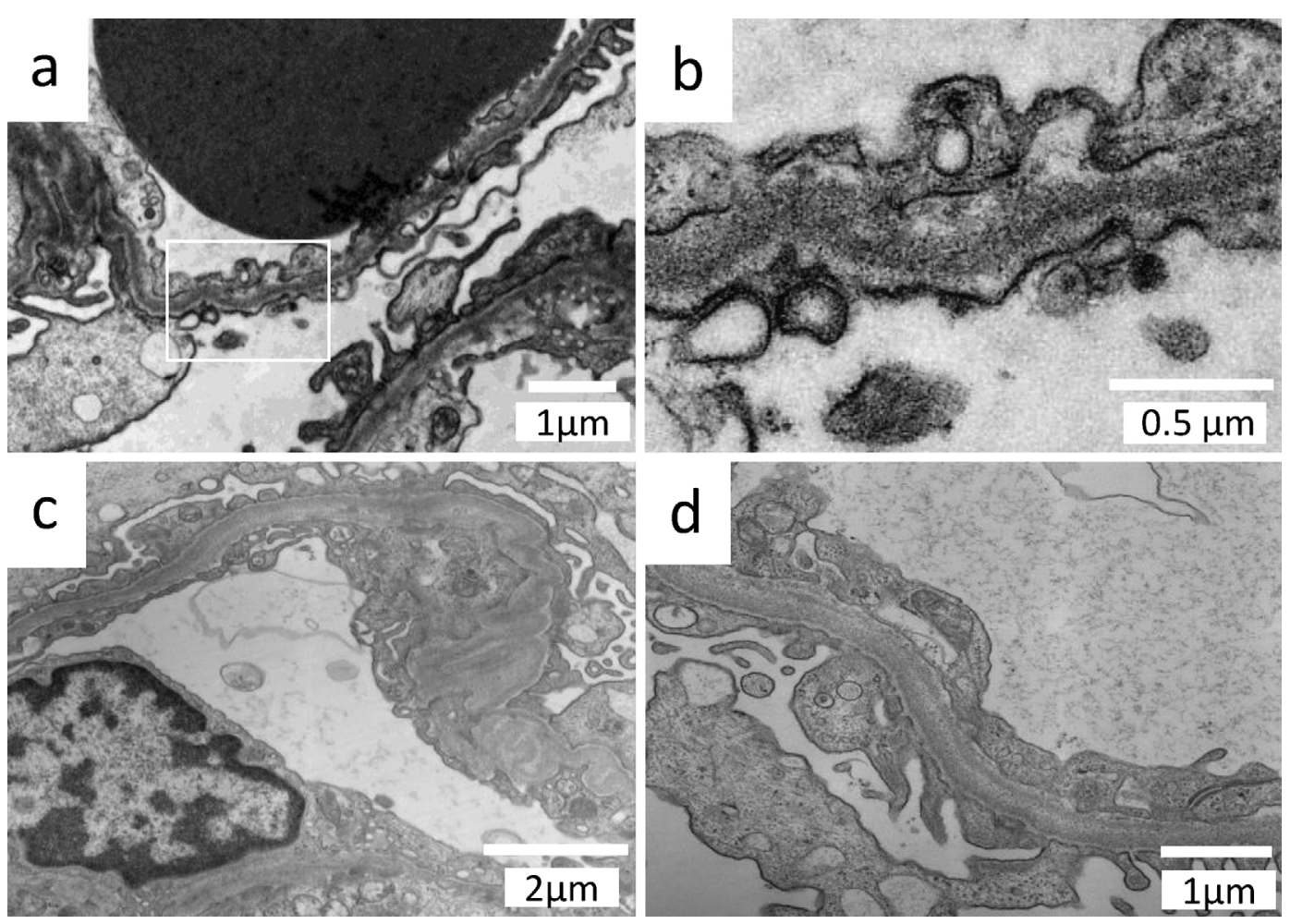

Figure 2. Electron micrographs of the first (a, b) and second (c, d) renal biopsy specimens. (a) Diffuse thinning of the glomerular basement membrane (GBM) is observed $(\times 5,000)$. (b) High-power field image of the square in a. (c) Irregular thinning, thickening, wrinkling, and collapse in the GBM are observed $(\times 12,000)$. (d) Irregular lamella densa in the $\mathrm{GBM}$ is visible $(\times 30,000)$.

we were unable to exclude the possibility of ADAS.

One of the diagnostic criteria for AS is an abnormality in type IV collagen staining. In contrast, TBMN is normally diagnosed by thinning of the GBM, without the assessment of type IV collagen staining. In our case, the initial diagnosis of TBMN was based on the ultrastructural features of thinning GBM without thickening, wrinkling, reticulation, or lamellation. Type IV collagen staining on the first biopsy specimen indicated that the $\alpha 5$ (IV) expression was reduced without any apparent increase in $\alpha 2$ (IV) expression in the GBM, showing a partial mosaic pattern (Fig. 3f). The extent of these changes was more marked on the second biopsy (Fig. 3i). Based on our findings, we speculate that abnormality in type IV collagen staining appears earlier than abnormalities in ultrastructural features of the GBM.

The missed diagnosis of AS might have resulted from various factors. TBMN is diagnosed based on an observation of thinning in $50 \%$ or more of the GBM, as revealed on electron microscopy, but with no thickening, wrinkling, reticulation, nor lamellation in the GBM (3). However, the presence of diffuse GBM thinning is the only finding in $10 \%-20 \%$ of patients with AS, particularly in children (8). Notably, focal thinning and mild irregularity of the GBM are the only findings observed in a six-week-old XLAS model mouse with a COL4A5 mutation R471 X (9). Based on these previous reports, TBMN may simply be the clinical presentation of early stages of AS with slower disease progression. Our observation of attenuation of $\alpha 5(\mathrm{IV})$ staining without an apparent compensatory increase in $\alpha 2$ (IV) staining in the GBM on the first renal biopsy might be consistent with the ultrastructural findings of thinning of the GBM in the early stages of AS (8). Pathologically, in AS, a decrease in the $\alpha 5$ (IV) expression initially leads to thinning of the GBM, with a subsequent compensatory increase in $\alpha 1$ (IV) and $\alpha 2$ (IV), which are normally only expressed in the GBM during the embryonic period. This change might result in thickening, wrinkling, reticulation, and lamellation in the GBM. The slowly progressive AS in our case, described by a serial biopsy, is consistent with previous reports $(3,8)$.

Generally, in XLAS, patients with negative staining of $\alpha 5$ (IV) are considered to be typical, whereas patients with normal or attenuated staining of $\alpha 5$ (IV) are thought to be atypical. Among a cohort of 25 men with XLAS, negative $\alpha 5$ (IV) staining was reported in $40 \%$ (10). Staining of $\alpha 5$ (IV) in the GBM is typically negative in men with XLAS and all patients with ARAS (5), as the $\alpha 3-4-5$ collagen network does not form. However, even in cases of AS, there are some patients whose $\alpha 5$ (IV) expression is partially reduced. In these cases, we should consider three possible patterns of AS when making a diagnosis. The first is the pattern in women with XLAS with a normal or reduced expression of $\alpha 5$ (IV) due to an X-linked inactivation mechanism (11). The second is the pattern in men with XLAS, in whom $\alpha 5$ (IV) is incompletely produced due to a substitution of COLAA5, as well as ARAS patients with a substitution of COLAA3 or COL4A4. Notably, positive $\alpha 5(\mathrm{IV})$ staining has been identi- 

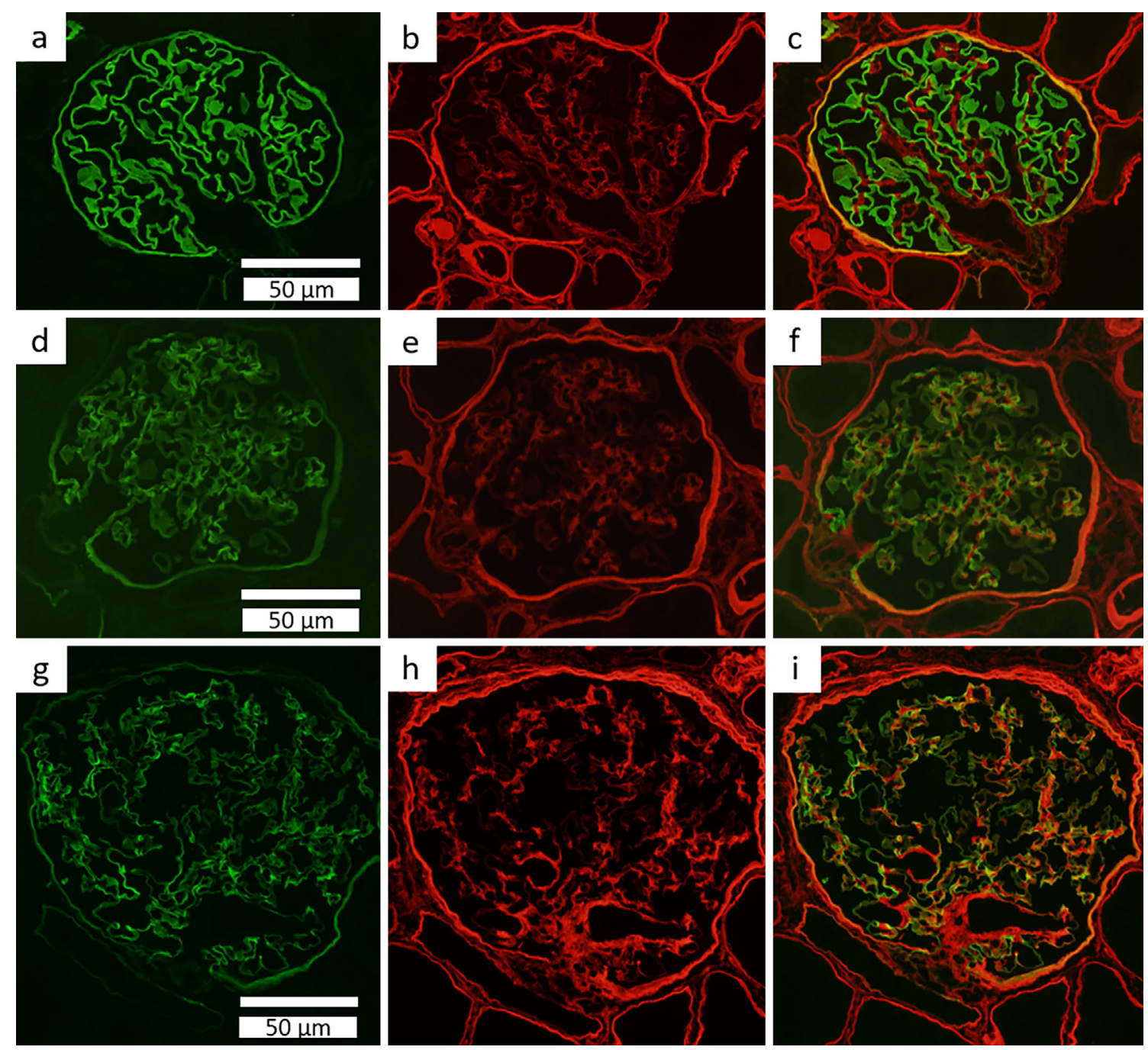

Figure 3. Immunohistochemical staining of type IV collagen. Type IV collagen staining in the control (a-c), in the first biopsy specimen (d-f), and in the second biopsy specimen (g-i); $\alpha 5$ (IV) staining (green) (a, d, g), $\alpha 2$ (IV) staining (red) (b, e, h), and merged images for $\alpha 5$ (IV) and $\alpha 2$ (IV) (c, f, i) (original magnification, $\times 200$ ). In a control kidney, $\alpha 5$ (IV) shows positive staining in the glomerular basement membrane (GBM) (a), and $\alpha 2$ (IV) shows positive staining in the mesangial region (b). In the first biopsy specimen, $\alpha 5$ (IV) shows partially reduced staining in the GBM (d), while $\alpha 2$ (IV) shows positive staining in mesangial region without an apparent increase in the GBM (e). In the second biopsy specimen, glomeruli were slightly hypertrophic (g-i). $\alpha 5$ (IV) shows partially reduced staining in the GBM, which is more obvious than at the first biopsy (g), with partially positive $\alpha 2$ (IV) staining in the GBM (h). In the first biopsy specimen, the merged image reveals a partial mosaic pattern in the GBM (f). In the second biopsy specimen, the merged image clearly reveals a mosaic pattern in the GBM (i).

fied in $\geq 20 \%$ of men with XLAS who exhibit a mild disease phenotype (12). Another study reported a reduced $\alpha 5$ (IV) expression in a patient with ARAS with a homozygous COL4A4 substitution who successfully delivered a child (13). The third is the pattern in patients with ADAS, in whom the $\alpha 5$ (IV) expression reduced. Previous reports have indicated that $\alpha 5(\mathrm{IV})$ is normal in patients with ADAS because one allele has a normal $\alpha 3$ (IV) or $\alpha 4$ (IV) expression (5). However, we recently reported a case in which $\alpha 5$ (IV) was reduced due to a heterozygous COL4A4 nonsense mutation (14). The formation of a network with sufficient type IV collagen requires $\alpha 3-4-5$ to maintain the function of the GBM. If the expression of $\alpha 3$ (IV) or $\alpha 4$ (IV) decreases, the expression of $\alpha 5$ (IV) will inevitably decrease as well. Although no mutation was identified, our patient may have had XLAS with a substitution or ADAS, based on his family history. Furthermore, partial attenuation of the $\alpha 5$ (IV) expression in Bowman's capsule was observed in our patient (Fig. 2d, 2f, $2 \mathrm{~g}$ and 2i). Since the $\alpha 5$ (IV) expression in Bowman's capsule is preserved in ADAS (14), our patient was presumed to have had XLAS with a substitution of COLAA5.

In conclusion, type IV collagen staining might be very useful for the early diagnosis of AS, especially in cases with 
GBM abnormalities for whom AS is a possibility.

\section{Author's disclosure of potential Conflicts of Interest (COI).}

Toshio Mochizuki: Employment, Otsuka Pharmaceutical Co, Chugai Pharmaceutical Co, Kyowa Hakko Kirin Co and JMS Co; Honoraria, Otsuka Pharmaceutical Co. Hiroshi Kataoka, Otsuka Pharmaceutical Co, Chugai Pharmaceutical Co, Kyowa Hakko Kirin Co and JMS Co.

\section{Funding statement}

None

\section{Study institution}

Department of Nephrology, Tokyo Women's Medical University, 8-1 Kawada-cho, Shinjuku-ku, Tokyo 162-8666, Japan

\section{Supplementary Material}

Supplementary Figure 1. The clinical course and medications after the first renal biopsy.

\section{References}

1. Hudson BG, Tryggvason K, Sundaramoorthy M, Neilson EG. Alport's syndrome, Goodpasture's syndrome, and type IV collagen. New Engl J Med 348: 2543-2556, 2003.

2. Nozu K, Nakanishi K, Abe Y, et al. A review of clinical characteristics and genetic backgrounds in Alport syndrome. Clin Exp Nephrol 23: 158-168, 2019.

3. Haas M. Alport syndrome and thin glomerular basement membrane nephropathy: a practical approach to diagnosis. Arch Pathol Lab Med 133: 224-232, 2009.

4. Voskarides K, Damianou L, Neocleous V, et al. COL4A3/COL4A4 mutations producing focal segmental glomerulosclerosis and renal failure in thin basement membrane nephropathy. J Am Soc Nephrol 18: 3004-3016, 2007.
5. Imafuku A, Nozu K, Sawa N, et al. Autosomal dominant form of type IV collagen nephropathy exists among patients with hereditary nephritis difficult to diagnose clinicopathologically. Nephrology 23: 940-947, 2018.

6. Yamamura T, Nozu K, Fu XJ, et al. Natural history and genotypephenotype correlation in female X-linked Alport syndrome. Kidney Int Reports 2: 850-855, 2017.

7. Horinouchi T, Nozu K, Yamamura T, et al. Detection of splicing abnormalities and genotype-phenotype correlation in X-linked Alport syndrome. J Am Soc Nephrol 29: 2244-2254, 2018.

8. Heidet L, Gubler MC. The renal lesions of Alport syndrome. J Am Soc Nephrol 20: 1210-1215, 2009.

9. Hashikami K, Asahina M, Nozu K, Iijima K, Nagata $M$, Takeyama M. Establishment of X-linked Alport syndrome model mice with a Col4a5 R471X mutation. Biochem Biophys Rep 17: 81-86, 2019.

10. Said SM, Fidler ME, Valeri AM, et al. Negative staining for COL 4A5 correlates with worse prognosis and more severe ultrastructural alterations in males with Alport syndrome. Kidney Int Reports 2: 44-52, 2017.

11. Jais JP, Knebelmann B, Giatras I, et al. X-linked Alport syndrome: natural history in 195 families and genotype-phenotype correlations in males. J Am Soc Nephrol 11: 649-657, 2000.

12. Hashimura Y, Nozu K, Kaito H, et al. Milder clinical aspects of $\mathrm{X}$-linked Alport syndrome in men positive for the collagen IV alpha5 chain. Kidney Int 85: 1208-1213, 2014.

13. Nishizawa $Y$, Takei $T$, Miyaoka $T$, Kamei $D$, Mochizuki $T$, Nitta $\mathrm{K}$. Alport syndrome and pregnancy: good obstetric and nephrological outcomes in a pregnant woman with homozygous autosomal recessive Alport syndrome. J Obstet Gynaecol Res 42: 331-335, 2016.

14. Akihisa T, Sato M, Wakayama Y, et al. Glomerular basement membrane protein expression and the diagnosis and prognosis of autosomal dominant Alport syndrome. Kidney Med 1: 391-396, 2019.

The Internal Medicine is an Open Access journal distributed under the Creative Commons Attribution-NonCommercial-NoDerivatives 4.0 International License. To view the details of this license, please visit (https://creativecommons.org/licenses/ by-nc-nd/4.0/).

(C) The Japanese Society of Internal Medicine Intern Med Advance Publication 